\title{
Does intraoperative fluoroscopic 3D imaging provide extra information for fracture surgery?
}

\author{
B. Carelsen $\cdot$ R. Haverlag $\cdot$ D. Th. Ubbink $\cdot$ \\ J. S. K. Luitse · J. C. Goslings
}

Received: 21 September 2007 / Published online: 13 September 2008

(C) The Author(s) 2008. This article is published with open access at Springerlink.com

\begin{abstract}
Fracture surgery of the extremities using 2D fluoroscopy frequently fails to detect the suboptimal positioning of implants and joint incongruities. The use of intraoperative 3D-rotational X-ray (3D-RX) imaging with a new $\mathrm{X}$-ray device potentially reveals these failures. We compared 50 intraoperative (2D) results of surgery and certainty about the effectiveness of different aspects of fracture reduction as interpreted from conventional (2D) methods versus intraoperative 3D-RX in 42 distal extremity fractures by means of a surgery questionnaire. In addition, we investigated the need for revision surgery based on postoperative radiological findings in 81 patients. After fracture reduction, just before a 3D-RX scan, the surgeon preoperatively assessed the result of surgery. Three months after surgery, the 3D-RX scan was judged by three experienced surgeons independently. Intraoperative 3D-RX showed significantly more information as to screw positioning and rotation of the fracture reduction than the conventional
\end{abstract}

The Medical Ethics commission of the Academic Medical Center, University of Amsterdam has approved "Evaluation of the clinical merit and image quality of intraoperative 3D-RX imaging for fracture surgery of the wrist, elbow and ankle."

B. Carelsen $\cdot$ R. Haverlag $(\varangle) \cdot$ D. Th. Ubbink ·

J. S. K. Luitse · J. C. Goslings

Department of Medical Physics,

Trauma Unit of the Department of Surgery,

Suite G7-250, Academic Medical Center,

University of Amsterdam, Meibergdreef 9,

1105AZ Amsterdam ZO, The Netherlands

e-mail: r.haverlag@amc.uva.nl

D. Th. Ubbink

Department of Surgery and Clinical Epidemiology,

Biostatistics \& Bioinformatics, Academic Medical Center,

University of Amsterdam, Amsterdam, The Netherlands method $(p<0.005)$. None of the 81 patients in whom 3D-RX was performed needed surgical revision based on postoperative radiological examinations. Intraoperative 3D-RX with this new device scanning offers additional information about extremity fracture reduction as compared to conventional intraoperative $2 \mathrm{D}$ imaging, and may reduce the need for revision surgery. The value of 3D-RX on functional outcomes still needs to be assessed.

Keywords Extremities - Trauma surgery ·

$\mathrm{X}$-ray $\cdot \mathrm{CT} \cdot 3 \mathrm{D}$

\section{Introduction}

With the use of conventional C-arm fluoroscopy in fracture surgery of the extremities, suboptimal positioning of implants (viz., screws, plates) and joint incongruities frequently remain unrevealed in clinical practice $[2,4,5,14$, $16,17]$. These imperfections are often only recognized on postoperative computed tomography (CT) scans $[2,4,12$, $14,21]$. AO guidelines recommend an anatomical reduction of the fracture as a basis for optimal outcome. The reported malreduction, redisplacement and complication rate for fracture surgery of the extremities based on radiological result can be up to $26 \%$ [3, 6, 9, 11, 19]. To obtain more intraoperative information, one possible solution could be surgical navigation based on preoperative CT. However, registration of small joints and multiple fragments as encountered in fracture surgery of the distal extremities is laborious and inaccurate [18]. Another solution to avoid unexpected and/or suboptimal postoperative radiological results is better intraoperative imaging.

Intraoperative use of 3-dimensional rotational X-ray (3D-RX) for fracture surgery of the extremities has been 
reported regarding the Arcadis Orbic $3 D$ in literature to lower revision in $21-39 \%$ of the cases $[8,12,14,15,20]$. Intraoperative revision of reduction, as indicated by the 3D scan, occurred for 11-19\%, and for implant position 11$26 \%$ of the operations in these studies [8, 14]. These authors did not register the pre-3D-scan status based on conventional examination and 2D fluoroscopy, but considered this to be optimal. However, intraoperative imaging is often used to achieve an optimal result in a stepwise and minimally invasive manner. Therefore, instant optimal results and confidence regarding these results are not selfevident, that is, intraoperative 3D-RX scanning guides the different stages of an operation. The threshold for revision surgery is much higher when the patient has left the OR. Small imperfections that were revealed with aid of intraoperative 3D-RX could have been revised directly, but less likely afterwards. Further investigation of revisions avoided is desired as well as of revision rates during follow-up.

All above referenced publications used the Arcadis Orbic 3D (Siemens Healthcare, Erlangen, Germany), we used a recently new introduced mobile C-arm with 3D-RX capabilities, the BV Pulsera with 3D-RX (Philips Healthcare, Best, The Netherlands).

Our research objective was to compare performance and certainty about the procedure result as interpreted from conventional methods versus additional intraoperative 3D$\mathrm{RX}$ for fracture reduction of the extremities with this new device. We hypothesized that 3D imaging would reveal a different, less satisfying surgical performance, but with more certainty about the procedure result. Subsequently, when acted upon these findings during the same surgical procedure, we expect a low revision rate because suboptimal reduction and fixation are detected intraoperatively and directly adjusted.

\section{Materials and methods}

All consecutive patients treated for intra-articular fractures of the foot, wrist, elbow or shoulder, between August 2005 and December 2006 in the Academic Medical Center (a tertiary referral center) at the University of Amsterdam were eligible for inclusion. Final inclusion with additional application of the 3D-RX system depended on the availability of the system, practical accessibility of the scanning area by the $\mathrm{C}$-arm and a fracture type according to an AO classification of B2 or higher. Approval of the medical ethics board for this study was obtained, and all patients signed an informed consent.

Surgical procedures were performed according to standard protocols. For ankle and foot surgery, a standard table (AlphaMaquet $^{\circledR}$, Maquet, Baambrugge, NL) was used. As long as the foot is not in line with the table side rails no
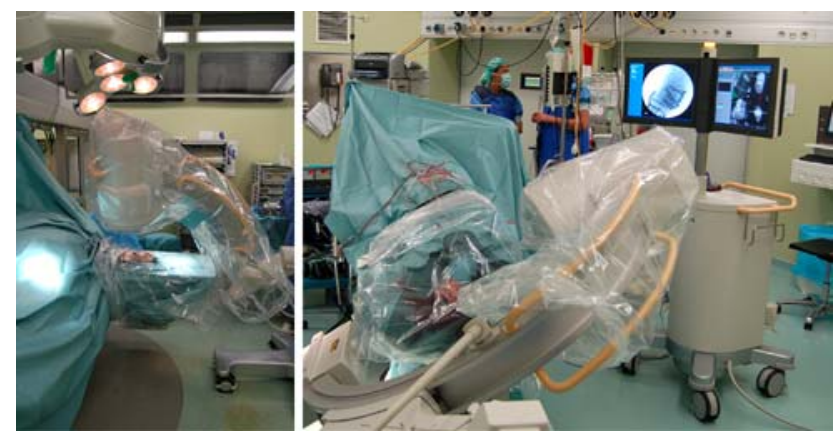

Fig. 1 Left panel: a picture of the 3D-RX scanning setup during a distal radius fracture operation of the wrist. Right panel: the scanning of a calcaneal fracture. Both extremities are placed on a carbon fiber rest inside a sterile bag

metal artifacts will appear (Fig. 1, right). For hand and wrist surgery, we utilized a RADIUS table (Liftac AG, Zurich, Switzerland), which is a unilateral suspended-arm table with a carbon fiber tabletop without ground support to allow rotational imaging (Fig. 1, left panel). Before positioning the $\mathrm{C}$-arm, a sterile bag (such as normally used to cover the image intensifier or an instrument table) was placed over the operation zone by the surgeon and instrumenting nurse. Thus, the extremity, table and drapes are inside the bag to keep the operation zone sterile. An anteroposterior and lateral low-dose X-ray image was taken to confirm the alignment of the system to the patient's anatomy.

During the operation, the surgeon decided whether and when to perform a subsequent 3D-RX scan. If no scans were made during the operation, at least one was made at the end of the operation just before wound closure. The surgeon was free to check in any way the current operation state by means of 2D fluoroscopy or examination. After the scan was made, but before the 3D image was shown, the surgeon answered the questions used for intraoperative assessment of the operation result as shown in "Appendix A", Table 4. The first 50 scans were included in this assessment. No questionnaire was taken immediately after the 3D to avoid bias.

For 3D-RX imaging, the BV Pulsera (Philips Healthcare, Best, The Netherlands) with 3D-RX system was used. This is a new mobile $\mathrm{C}$-arm, modified for motorized movement and integrated with a Philips 3D-RA workstation. For this study, a series of 225 projection images are taken during a $200^{\circ}$ rotation of the C-arm in $30 \mathrm{~s}$, which is used to reconstruct a 3D-RX scan. The dose of each image in this scanning run is dynamically adjusted to obtain low dose and optimal image quality. The maximum effective dose for a hand/wrist 3D-RX scan is $6 \mu \mathrm{Sv}$, for a foot $10 \mu \mathrm{Sv}$ [12]. For comparison, for CT imaging of the lower extremities a typical dose is $120 \mu \mathrm{Sv}$ and the dose of plain hand and foot radiograph are $0.17 \mu \mathrm{Sv}$ and $0.7 \mu \mathrm{Sv}$, 
respectively $[7,13]$. Depending on the size of the anatomy of interest, we adjusted the magnification to either a spherical shape with a diameter of 12.5 or $18 \mathrm{~cm}$, with slices of 0.5 or $0.7 \mathrm{~mm}$ thick in all directions, respectively. After reconstruction, the 3D-RX data set could be viewed on a workstation in the operating room. Both volume rendering and coronal, sagittal and axial slices were available. After this, the operation could continue, while the images were being processed $(4 \mathrm{~min})$. Sterile coverage, aligning and scanning takes approximately $3 \mathrm{~min}$, in total $7 \mathrm{~min}$ are needed to obtain a 3D image. Next, the surgeon examined the 3D-RX images and continued or finished the operation.

Four months after obtaining the first 50 scans, three experienced trauma surgeons, who also operated the majority of the patients included, reviewed the 3D-RX images. The reviewers were offered the images in a blinded and random fashion and were asked to answer the questions regarding the intraoperative assessment of the operation result. Primary outcome was the result of pre-3D scan surgery as judged by the surgeon, based on conventional examination and 2D fluoroscopy, in comparison with the judgment based on the 3D-RX scan by means of the questionnaire. Secondary outcome was the number of revision surgeries based on postoperative radiological findings like radiographs or CT imaging, for patients treated with the help of 3D-RX. Record was kept for the number of revision surgery procedures within 3 months of follow-up, based on the radiological outcomes.

The judgment results from the three reviewers were averaged and are presented as median values. A MannWhitney $U$ test was used to analyze differences between the surgeon's answer and the median value of the three reviewers for every question ("Appendix A", Table 4). A two-tailed significance level of $p<0.05$ was considered significant.

Based on the literature findings, a $20 \%$ difference in revision rates can be expected when 3D-RX imaging is used
$[8,14]$. This difference was used to calculate a minimum number of 14 patients required to achieve statistical significance: if for three out of 14 patients $(21.4 \%)$ a revision is avoided, this outcome has a $95 \%$ confidence interval of $5-51 \%$, that is., the revision rate is significantly above 0 . We included a larger number of patients to be able to increase the precision of the point estimates also regarding other outcomes.

To analyze the agreement among the three reviewers regarding their judgment of the 3D-RX scans, we determined the percentage of agreement (i.e., the accuracy) among the surgeons within one point on the 5-point scale. This was also used to assess the intra-observer agreement. For this purpose, three patients (a hind foot, an ankle and a wrist fracture) were reviewed a second time by all reviewers six weeks later.

\section{Results}

A total of 81 operations concerning 81 patients were operated with aid of 3D-RX and included in this study. In total, 97 3D-RX scans were performed, 65 patients were scanned once, 16 patients twice, during different stages of the operation (on average 1.2 scans per patient). The average operation time was $126 \mathrm{~min}$. The mean age of the group was 44 years (ranging from 12 to 86) and comprised 45 males and 36 females. Patient data are shown in Table 1. Excluded 3D-RX scans were those with a poor image quality due to wrong aligning (3), too much metal (3), or inexplicable failure (2).

The comparison between conventional methods and intraoperative 3D-RX scanning for fracture surgery of the extremities showed a significantly lower performance as to screw positioning and rotation of the fracture reduction after 3D-RX $(p<0.002$, Table 2$)$. The same trend could be observed for the other aspects interrogated. Certainty about the rotation of the fracture reduction performance was
Table 1 Description of the study population. Numbers between parenthesis indicate the number of patients included in the questionnaire

${ }^{a}$ In one case after removal of osteosynthesis material a $3 \mathrm{D}-\mathrm{RX}$ scan was made and on the basis of the image no further intervention was done

\begin{tabular}{lllll}
\hline Extremity & Patients & Scans & Classification & Scans \\
\hline Foot & $52(24)$ & $66(31)$ & Hind foot & $26(12)$ \\
& & & Ankle & $30(16)$ \\
& & Forefoot & $5(2)$ \\
& & Arthrodesis & $4(1)$ \\
& & & Perioperative imaging & $1^{\mathrm{a}}$ \\
Wrist & $25(16)$ & $27(17)$ & Distal radius & $23(14)$ \\
& & & Osteosynthesis carpal/metacarpal fracture & $4(3)$ \\
Elbow & $3(1)$ & $3(1)$ & Radial head & $3(1)$ \\
Shoulder & $1(1)$ & $1(1)$ & Scapula & $1(1)$ \\
Total & $81(42)$ & $97(50)$ & & \\
\hline
\end{tabular}


Table 2 Questionnaire results of the 50 scans investigated $N \quad S<R \quad S>R \quad$ Significance $(p)$

Performance

$\begin{array}{lllll}\text { Positioning K-wires? } & 14 & 1 & 6 & 0.12 \\ \text { Positioning screws? } & 34 & 4 & 20 & 0.0004^{*} \\ \text { Reduction fracture: alignment? } & 47 & 11 & 16 & 0.09 \\ \text { Reduction fracture: rotation? } & 36 & 4 & 18 & 0.002^{*} \\ \text { Joint surface? } & 48 & 12 & 22 & 0.1 \\ \text { Certainty } & & & & \\ \text { Positioning K-wires? } & 14 & 6 & 4 & 0.6 \\ \text { Positioning screws? } & 34 & 14 & 7 & 0.09 \\ \text { Reduction fracture: alignment? } & 47 & 13 & 15 & 0.9 \\ \text { Reduction fracture: rotation? } & 36 & 17 & 10 & 0.05^{*} \\ \text { Joint surface? } & 48 & 17 & 19 & 0.9\end{array}$

$N$ is the total number of times the question was answered or applicable. $S$ is the intraoperative surgeons score, $R$ the median of the reviewers. $S>R$ concerning the performance means that the reviewers are less satisfied with the performance of the surgeon before the 3D-RX scan than her/himself. $S>R$ concerning the certainty question means that reviewers are less certain about the diagnosis than the surgeon before the 3D-RX scan about his/her performance

Values in * represents a significant difference

significantly larger $(p<0.05)$ if based on 3D-RX images (Table 2).

Inter-observer accuracy was good $(85 \pm 2 \%)$ for a difference of up to one point on the 5-point scale, while $40 \pm 4 \%$ was scored unanimously. Intra-observer accuracy was excellent $(94 \pm 3 \%)$ for a difference of up to one point between the first and second judgment, while $62 \% \pm 3 \%$ was scored unanimously.

None of the 81 patients included in this study needed revision based on the postoperative radiological examinations. An example of 3D-RX imaging versus 2D fluoroscopy is shown in Fig. 2.

\section{Discussion}

Fracture surgery of the extremities with conventional examination methods frequently misses out suboptimal positioning of implants and joint incongruities during the operation. These problems remain unrevealed until postoperative radiography or CT imaging is performed $[2,4,14$ $16,21]$

This study was performed with a new device for intraoperative 3D-RX imaging. The main differences between a longer known X-ray device, the Arcadis Orbic 3D (Siemens Healthcare, Erlangen, Germany) are shown in Table 3. The 3D movement of the Arcadis is able to reach more parts of the body. According to technical specifica-
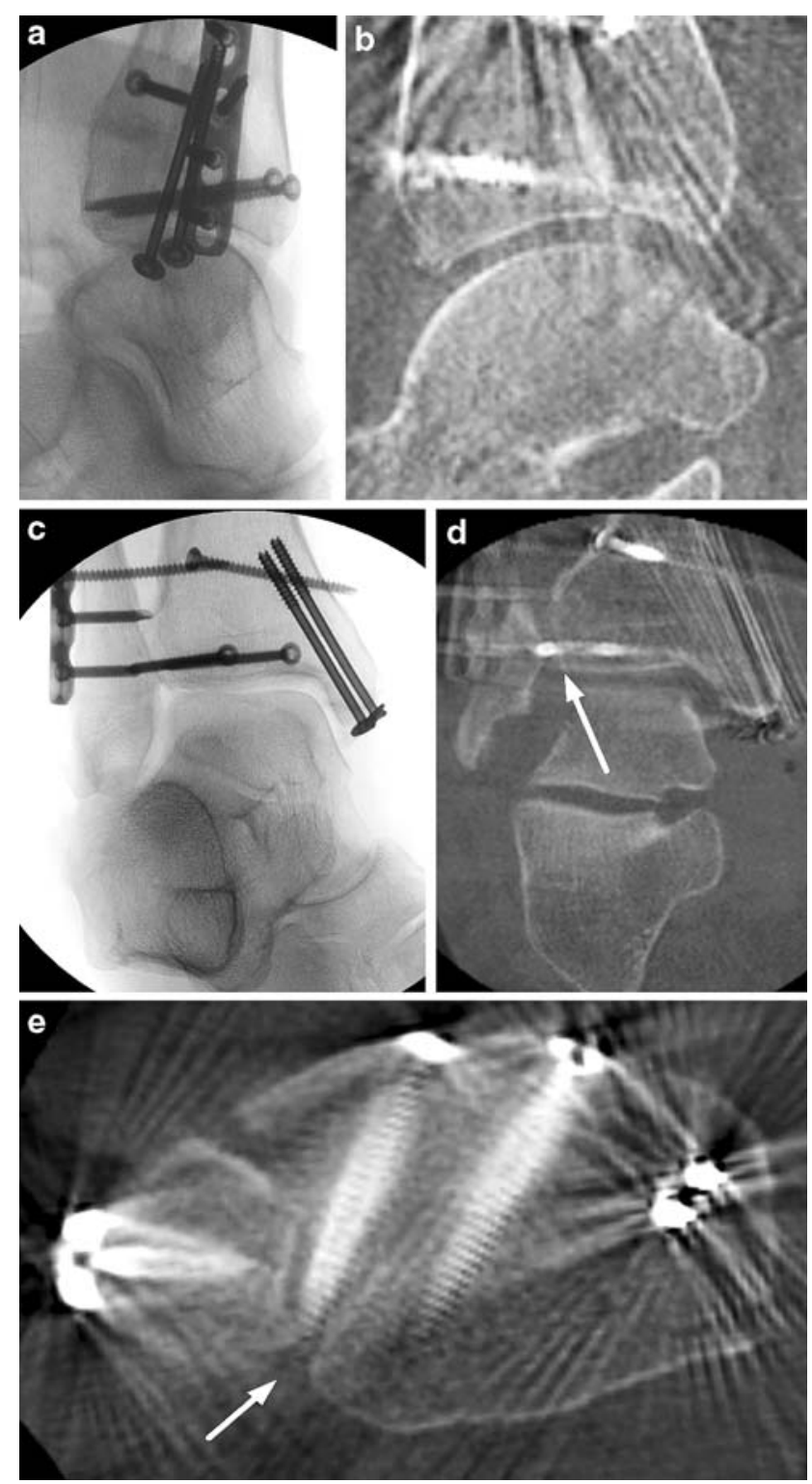

Fig. 2 Intraoperative $X$-ray images of patient treated minimally invasive for an AO type $\mathrm{C} 2$ trimalleolar ankle fracture. Shown are the fluoroscopy images (a, c) and 3D-RX slices in respectively sagittal, coronal and axial direction $(\mathbf{b}, \mathbf{d}, \mathbf{e})$. In the coronal 3D-RX slice it is clearly visible that a screw penetrates the distal tibio-fibular joint (arrow in image $\mathbf{d}$ and $\mathbf{e}$ )

tions, the Pulsera should at least provide the comparable image quality.

Limitations of this study include patient selection. Although we prospectively included all patients, the scan movement was not predefined. However, a protocol to scan at predefined moment is cumbersome in patients suffering from a great variety of fractures qualifying for 3D-RX scanning. In addition, 3D-RX scanning may be inconclusive, which occurred in $8 \%$, of the scans in our study. However, these occurred in the first 58 consecutive patients (14\%). Improved experience of the radiology technicians 
Table 3 Overview of relevant properties which clearly are different for both systems

\begin{tabular}{lll}
\hline Property & $\begin{array}{l}\text { BV Pulsera } \\
\text { with 3D-RX }\end{array}$ & $\begin{array}{l}\text { Arcadis } \\
\text { Orbic 3D }\end{array}$ \\
\hline 3D movement & Head or extremity & $\begin{array}{l}\text { Lateral } \\
\text { Duration of scan }\end{array}$ \\
No. of images/scan & $90 \mathrm{~s}$ & $60 \mathrm{~s}$ or $120 \mathrm{~s}$ \\
Kind of X-ray images & Pulsed $(10 \mathrm{~ms})$ & $50-100$ \\
II magnifications & $6^{\prime \prime}-9^{\prime \prime}-12^{\prime \prime}$ & Fluoroscopy \\
X-ray generator power & $15 \mathrm{~kW}$ & $6^{\prime \prime}-9^{\prime \prime}$ \\
\hline
\end{tabular}

and anticipating metal artifacts avoided problems in the remaining scans.

Furthermore, the golden standard as to the effectiveness of fracture surgery is long-term functional outcome. However, examination of CT scans or postoperative 2D radiographs is considered an acceptably predictive intermediate-term outcome measure $[3,6,9,11,19]$. Currently, there is no golden standard available during surgery, which hampers clinical evaluation of this technique which is a weakness of our study. Evaluation of the radiological diagnostic value of 3D-RX has been done previously and was considered adequate and similar to $\mathrm{CT}$ and radiography for imaging bony structures $[1,8,10,12,14,15,20]$. We compared 3D-RX with the conventional intraoperative practice, which is known to have shortcomings [2, 4, 12, 14, 21]. Thus, the particular shortcomings could be exposed. In this manner, we proved that intraoperative 3D imaging provides additional information, an import condition to further investigate long-term functional patient outcome.

Since no general classification system exists to rate radiological outcomes of fracture surgery, we designed our own, based on the standard way surgeons and radiologists examine radiographs and/or CT scans. The questionnaire gives a comprehensive judgment about the results of the surgical procedure. The intra- and inter-observer accuracy of this study was good. Nevertheless, the questionnaire should be validated to allow comparison of the results with those from other centers.

This study showed that intraoperative 3D-RX provides extra information to the surgeon, especially regarding the assessment of rotation of fracture reduction and screw placement. These are the two important performance parameters that are hard to appreciate by conventional examination and fluoroscopic inspection. Especially with fluoroscopy, the angle of imaging and over-projection can lead to unreliable results. With intraoperative 3D-RX, images can be viewed from every possible angle without extra fluoroscopy time, although metal artifacts may distort the images. Our results showed that $18 \%$ of the screw positionings at least two points less satisfying were rated on the
3D-RX images than expected intraoperatively. If we consider this condition a revision it is between the results of Richter et al. (26\%) and Hufner et al. (10.3\%) [8, 14]. Also, if we consider inclusion time and number for this study a revision rate of $16 \%$ or higher is required in order to be cost-beneficial based on Hufner et al. [8]. Moreover, during the course of this study we experienced that the frequency of use increased and the device is shared with other disciplines as well. Nevertheless, it should be stressed that most intraoperative failures are revised more frequently than when revealed postoperatively.

In this study, the rotation of the reduction was diagnosed with more certainty by the reviewers than the surgeon without the 3D-RX scan. Except for the diagnosis of the alignment of the reduction and the joint surface the reviewers were more certain about their diagnosis than the surgeon. This (un)certainty, however, does not only appreciate the result of surgery, but also the character of the surgeons involved. Moreover, the 'noisy' 3D-RX images do not provide such confidence as obtained by $\mathrm{CT}$ and radiography.

In this study no revisions were indicated on the basis of postoperative radiological results, which can be attributed to the fact that 3D-RX imaging was already in use. The reported radiology-based failure rate, if treated conventionally, is higher and ranges from $10 \%$ in distal radius fractures to over $26 \%$ in calcaneus or malleolus tertius fractures $[6,9]$. These failures would be, at least in most cases, a strong indication for revision surgery.

\section{Conclusion}

Intraoperative 3D-RX clearly provides more information about the result of fracture reduction procedures than conventional 2D-scanning. The reduction in indications for revision surgery after a relatively short follow-up period is promising, but not equivalent to a good functional outcome. This patient-relevant parameter still needs to be investigated after a longer follow-up period which is meaningful as proven by this study. In our hospital, the use of intraoperative 3D-RX imaging has become a standard procedure during surgical treatment of complex joint fractures of the extremities.

Acknowledgments The authors wish to thank K. J. Ponsen and G. J. Streekstra of the AMC, Amsterdam, and P. van Kemenade of Philips Healthcare, Best, for their contribution to this study.

Conflict of interest statement The $\mathrm{PhD}$ project of B.C. is funded by Philips Healthcare and the Dutch ministry of economical affairs.

Open Access This article is distributed under the terms of the Creative Commons Attribution Noncommercial License which permits any noncommercial use, distribution, and reproduction in any medium, provided the original author(s) and source are credited. 


\section{Appendix A}

Table 4 Questions used for intraoperative assessment of the operation state and review

\begin{tabular}{lll}
\hline Question & Range performance & Range certainty \\
\hline Positioning K-wires? & 1. Redo & 1. Not \\
& 5. Satisfied & 5. Sure \\
Positioning screws? & 1. Redo & 1. Not \\
& 5. Satisfied & 5. Sure \\
Reduction fracture: & 1. Redo & 1. Not \\
alignment? & 5. Satisfied & 5. Sure \\
Reduction fracture: & 1. Redo & 1. Not \\
rotation? & 5. Satisfied & 5. Sure \\
Joint surface? & 1. Redo & 1. Not \\
& 5. Satisfied & 5. Sure \\
\hline
\end{tabular}

\section{References}

1. Carelsen B, Bakker NH, Boon SN, Fokkens WJ, Freling NJ, Noordhoek N (2004) Mobile 3D rotational X-ray: comparison with CT in sinus surgery. Medica Mundi 48:4-9

2. Catalano LW III, Barron OA, Glickel SZ (2004) Assessment of articular displacement of distal radius fractures. Clin Orthop Relat Res 423:79-84

3. Chen SH, Wu PH, Lee YS (2007) Long-term results of pilon fractures. Arch Orthop Trauma Surg 127:55-60

4. Edwards CC II, Haraszti CJ, McGillivary GR, Gutow AP (2001) Intra-articular distal radius fractures: arthroscopic assessment of radiographically assisted reduction. J Hand Surg 26:1036-1041

5. Euler E, Wirth S, Linsenmaier U, Mutschler W, Pfeifer KJ, Hebecker A (2001) Comparative study of the quality of C-arm based 3D imaging of the talus. Unfallchirurg 104:839-846

6. Handoll HH, Madhok R (2003) Surgical interventions for treating distal radial fractures in adults. Cochrane database of systematic reviews (Online).CD003209

7. Huda W, Gkanatsios NA (1998) Radiation dosimetry for extremity radiographs. Health Phys 75:492-499
8. Hufner T, Stubig T, Gosling T, Kendoff D, Geerling J, Krettek C (2007) Cost-benefit analysis of intraoperative 3D imaging. Unfallchirurg 110:114-121

9. Kapoor H, Agarwal A, Dhaon BK (2000) Displaced intra-articular fractures of distal radius: a comparative evaluation of results following closed reduction, external fixation and open reduction with internal fixation. Injury 31:75-79

10. Kotsianos D, Wirth S, Fischer T, Euler E, Rock C, Linsenmaier U, Pfeifer KJ, Reiser M (2004) 3D imaging with an isocentric mobile C-arm comparison of image quality with spiral CT. Eur Radiol 14:1590-1595

11. Langenhuijsen JF, Heetveld MJ, Ultee JM, Steller EP, Butzelaar RM (2002) Results of ankle fractures with involvement of the posterior tibial margin. J Trauma 53:55-60

12. Meier R, Kfuri M Jr, Geerling J, Hufner T, Krimmer H, Krettek C (2005) Intraoperative three-dimensional imaging with an isocentric mobile C-arm at the wrist. Handchir Mikrochir Plast Chir 37:256-259

13. MTA (2006) Calculated using 'CT-Dose' (tissue and effective dose software). http://www.mta.au.dk

14. Richter M, Geerling J, Zech S, Goesling T, Krettek C (2005) Intraoperative three-dimensional imaging with a motorized mobile $\mathrm{C}$ arm (SIREMOBIL ISO-C-3D) in foot and ankle trauma care: a preliminary report. J Orthop Trauma 19:259-266

15. Rubberdt A, Feil R, Stengel D, Spranger N, Mutze S, Wich M, Ekkernkamp A (2006) The clinical use of the ISO-C(3D) imaging system in calcaneus fracture surgery. Unfallchirurg 109:112-118

16. Ruch DS, Vallee J, Poehling GG, Smith BP, Kuzma GR (2004) Arthroscopic reduction versus fluoroscopic reduction in the management of intra-articular distal radius fractures. Arthroscopy 20:225-230

17. Sanders R, Fortin P, DiPasquale T, Walling A (1993) Operative treatment in 120 displaced intraarticular calcaneal fractures. Results using a prognostic computed tomography scan classification. Clinical Orthop Relat Res 290:87-95

18. Schep NW, van Vugt AB (2006) Navigation surgery and fracture treatment. Nederlands tijdschrift voor geneeskunde 150:23012306

19. Stulik J, Stehlik J, Rysavy M, Wozniak A (2006) Minimally-invasive treatment of intra-articular fractures of the calcaneum. J Bone Joint Surg Br 88:1634-1641

20. Wich M, Spranger N, Ekkernkamp A (2004) Intraoperative imaging with the ISO C(3D). Der Chirurg; Zeitschrift fur alle Gebiete der operativen Medizen 75:982-987

21. Zwipp H, Dahlen C, Randt T, Gavlik JM (1997) Complex trauma of the foot. Der Orthopade 26:1046-1056 